\title{
La construcción de valores en la educación: un reto
}

\section{Building values in education: a challenge}

\author{
Yohana Maricela Yaguana Castillo ${ }^{1 *}$, Xiomara Paola Carrera Herrera ${ }^{1}$ y Miury Marieliza Placencia Tapia ${ }^{1}$ \\ ${ }^{1}$ Universidad Técnica Particular de Loja \\ *jmyaguana@utpl.edu.ec
}

DOI: https://doi.org/10.26871/killkana_social.v3i2.469

\begin{abstract}
Resumen
La UNESCO considera a la inclusión y el respeto a la diversidad como componentes fundamentales de la convivencia armónica superponiéndose y eliminando la exclusión. Por ello, estamos llamados todos los que formamos parte del sistema educativo a desarrollar mecanismos generadores de confianza en sus miembros. La Universidad Técnica Particular de Loja desarrolla actividades complementarias a lo académico e investigativo como parte de la vinculación con la colectividad. Esto nos permite integrar funciones universitarias con la sociedad convirtiendonos en un centro de apoyo en la consecución de sus objetivos. Dichas actividades se identifican por ser esenciales, multidisciplinarias e integradoras, haciendo que la comunidad participa del acervo científico, tecnológico y humanístico, mediante la realización de un diagnóstico previo de las necesidades colectivas. Es así que surge el proyecto de vinculación denominado "La escuela en la educación de valores "el mismo que se desarrolló con la participación de 11 estudiantes y 2 docentes de Ciencias Humanas y Religiosas por medio del componente Practicum 3.2, objetivo es fortalecer estrategias metodologías que motiven el proceso de enseñanza aprendizaje en la formación de valores en los estudiantes, docentes y padres de familia de las instituciones educativas. En este estudio se utilizó una metodología cuantitativa, en él se aplicó una encuesta "La escuela en la educación de valores", a 290 adolescentes de 8 establecimientos educativos de las ciudades de Santa Rosa, Guayaquil, Quito Bahía de Caraqués, Cuenca Ibarra y Ambato. Así se logró promover la transferencia e intercambio de aprendizaje entre los miembros de la universidad y la comunidad, permitiendo el acercamiento de los estudiantes a un contexto real a través de talleres dinámicos y creativos que generaron impacto positivo especialmente en grupos vulnerables de la sociedad. La vivencia de valores está estrechamente relacionada con tres aspectos: convivencia escolar, las relaciones con el docente y en el hogar.
\end{abstract}

Palabras clave: valores, educación, desafío, estudiantes.

\begin{abstract}
The United Nations Educational, Scientific and Cultural Organization, UNESCO (acronym in Spanish) considers inclusion and respect for diversity as fundamental components of the harmonious coexistence as they overcome and eliminate exclusion. Therefore, all those belonging to the educational system are expected to develop procedures that can generate trust in its members. In addition to the academic and research activities; the Universidad Tècnica Particular de Loja develops complementary activities as part of the relationship with the community facilitating their interaction and becoming the support center in the achievement of its objectives. These activities are considered essential, multidisciplinary and integrative. They enable the community participation in the scientific, technological and humanistic heritage, through the prior diagnosis of the community needs. This way, the relationship project "The school in values education" started. This project was developed with the participation of 11 students and 2 Human and Religious Sciences' teachers, through the implementation of the 3.2 Practicum component. The aim was to strengthen strategies and methodologies in order to motivate the teaching-learning process on values formation for the educational institution's students, teachers, and parents. In this study, a quantitative methodology was used with the application of the survey, "The school in values education", to 290 adolescents from eight different educational establishments in the cities of Santa Rosa, Guayaquil, Quito, Bahía de Caraques, Cuenca, Ibarra, and Ambato. Thus, it was possible to promote learning transferring and exchange between the university and the community members; allowing to familiarize students with real contexts through dynamic and creative workshops that are generators of positive impact, especially on the most vulnerable groups of society. Experiencing values is closely related to three aspects: school coexistence, students-teacher relationships, and family relationships within the home.
\end{abstract}

Key words: values, education, challenge, students. 


\section{Introducción}

Hoy en día la vinculación de la Universidad con la sociedad permite un conjunto de acciones y procesos académicos ejecutados por todas las dependencias universitarias en cooperación con sectores externos a la Universidad, como el Estado, Instituciones Educativas, entro otras; orientados a resolver problemas y ejecutar programas y proyectos que tengan impactos positivos en el país y el mundo.

Esta aproximación de la universidad con la colectividad está fundada en la investigación, la transferencia de tecnología, extensión y en la responsabilidad social.

Uno de los desafíos actuales del docente es la práctica continua en valores dentro y fuera del aula, lo cualdespierta así el interés del educando hacia la vivencia continua de ellos. Una relación empática entre docentes y alumno será la plataforma para una efectiva transmisión de actitudes y habilidades sociales hacia la sociedad.

Muchos autores señalan la crisis de los valores morales en la sociedad actual, de ahí emerge la necesidad que se presenta en la sociedad moderna de una educación en valores que contemple algunos principios básicos para un desarrollo moral de la sociedad en que vivimos. Esto permitirá conducir a nuevas potencialidades para formar seres humanos respetuosos, autónomos, responsables y solidarios.

La educación en valores, desde la perspectiva de diferentes pedagogos, se concibe como propuestas pedagógicas un tanto diferente, los valores son designados como temas transversales, dado que fragmentan el currículum escolar en diferentes ámbitos de conocimiento y se conforman por contenidos temáticos de carácter interdisciplinar de trascendencia social. Por su parte, Jackson (1992) determina que los aprendizajes que tienen una estrecha relación con la esfera de valorar y actitudinal se consideran como un currículo oculto que está presente en la escuela como una forma de socialización y adaptación.

Es por ello, que para lograr en el aula una verdadera educación en valores, debe comprometerse a cumplir dos principales características: la primera debe estar fundamentada en el alumno, considerándole como persona vista desde la dignidad humana, investida de valores morales desde los que se potencian sus relaciones, creando un clima de respeto mutuo y tomando en cuenta las características particulares. La segunda implicaría vincular a la pedagogía con una fundamentación en la empatía y el afecto, asumiendo el sentido de la responsabilidad inherente al educador, así como satisfaciendo la necesidad de establecer mecanismos de colaboración entre padres y docentes, con la intensión de desarrollar la autonomía personal en el sentido crítico.

Es así que la Universidad Técnica Particular de Loja como parte de sus proyectos de vinculación con la colectividad fundamentada en la investigación, la transferencia de tecnología, extensión y en la responsabilidad social y con la participación de docentes del componente de Practicum 3.2 que se imparte en la titulación de Ciencias Humanas y Religiosas en conjunto con los estudiantes de la modalidad a distancia durante un año, realizaron un conjunto de acciones y procesos académicos ejecutados por todas las dependencias universitarias en cooperación con sectores externos a la Universidad, como son las instituciones educativas de algunas ciudades del país.

\section{Marco Teórico}

La educación sin duda debe estar al servicio de la realización de los objetivos más altos de la humanidad. Y como lo enuncian en el document, Populorum Progressio (1965) y en la Declaración sobre la Educación Cristiana, dichos objetivos fueron evidenciados con visión de futuro: apostar por el desarrollo armonioso de las capacidades físicas, morales e intelectuales, finalizadas a la gradual maduración del sentido de responsabilidad y la conquista de la verdadera libertad del ser humano. Desde esta perspectiva, se intuía que la educación debía estar al servicio de un nuevo humanismo, donde la persona social se encuentra dispuesta a dialogar y a trabajar para la realización del bien común.

Esta búsqueda de poder, humanizar desde la educación, sigue siendo actual. Así lo afirma Gordillo (2015), a pesar que las concepciones antropológicas basadas en el materialismo, el idealismo, el individualismo y el colectivismo, viven una fase de decadencia, todavía ejercen una cierta influencia cultural. A menudo ellas entienden la educación como un proceso de adiestramiento del individuo a la vida pública, en la que actúan las diferentes corrientes ideológicas, que compiten entre sí por la hegemonía cultural. En este contexto, la formación de la persona responde a otras exigencias: la afirmación de la cultura del consumo, de la ideología del conflicto, del pensamiento relativista, etc. Es necesario, por lo tanto, humanizar la educación; es decir, transformarla en un proceso en el cual cada persona pueda desarrollar sus actitudes profundas, su vocación y contribuir así a la vocación de la propia comunidad. El Papa Francisco definía el significado de "Humanizar la educación", como poner a la persona al centro de la educación, en un marco de relaciones que constituyen una comunidad viva, interdependiente, unida a un destino común. De este modo se cualifica el humanismo solidario.

De igual forma Suárez (2008), reafirma que no se limita a ofrecer un servicio formativo, sino que se ocupa de los resultados del mismo en el contexto general de las aptitudes personales, morales y sociales de los participantes en el proceso educativo. No solicita simplemente al docente enseñar y a los estudiantes aprender, más bien impulsa a todos a vivir, estudiar y actuar en relación a las razones del humanismo solidario. No programa espacios de división y contraposición, al contrario, ofrece lugares de encuentro y de confrontación para crear proyectos educativos válidos. Se trata de una educación —al mismo tiempo- sólida y abierta, que rompe los muros de la exclusividad, promoviendo la riqueza y la diversidad de los talentos individuales y extendiendo el perímetro de la propia aula en cada sector 
de la experiencia social, donde la educación puede generar solidaridad, comunión y conduce a compartirlo.

La UNESCO menciona sobre la inclusión y el respeto a la diversidad como componente fundamental de la convivencia armónica, superponiéndose y eliminando la exclusión. Por ello, se realiza un llamado a todos los que conforman el sistema educativo a desarrollar, tanto a nivel de estructuras, como de su cultura organizacional, mecanismos para generar confianza en sus miembros Las instituciones educativas tienen el potencial de transformarse en espacios que amplíen la capacidad creativa y afectiva de los seres humanos, y en lugar donde la confianza sea cultivada con el valor básico en el que tiene lugar los aprendizajes que a su vez se hacen extensivos a la familia y a la comunidad.

El estudio de los valores es un tema que permite observar al individuo desde sus conductas y actitudes. En el caso de la Filosofía, da lugar a una nueva ciencia de los valores o Axiología. La etimología de esta palabra procede de los términos griegos Axios (digno, valioso) y Logos (ciencia, tratado). Es fundamental señalar algunas de las numerosas definiciones del término Valor. Rokeach (1973) hace énfasis en la multidisciplinariedad de los valores señalando que "El concepto de valor (...) [es] capaz de unificar los aparentemente diversos intereses de todas las ciencias que se ocupan de la conducta humana"(p. 3).

Este trabajo nace desde el campo educativo donde se han destacado por aportes notables especificando la concepción del constructo valor. La educación tiene como función formar al ser humano desde su integralidad por lo que, como principio básico, reconoce a los educandos como personas, con una consolidación de los valores morales hacia una sociedad democrática. Se toma en consideración el término democrático como un modelo de equidad, libertad, honradez, solidaridad, armonía, humildad y respeto de pensamiento entre las personas.

En este sentido Ortiz (2003) hace referencia a la importancia de la educación y los valores como aspectos fundamentales de una sociedad:

"La educación es, por tanto, aquella actividad cultural que se lleva a cabo en un contexto intencionalmente organizado para la transmisión de los conocimientos, las habilidades y los valores que son demandados por el grupo social. Así, pues, todo proceso educativo está relacionado con los valores. Por medio de la educación, todo grupo humano tiende a perpetuarse, siendo los valores el medio que da cohesión al grupo al proporcionarles unos determinados estándares de vida" (p. 24).

Ruiz (2003) enfatiza cuatro funciones principales que desempeña la escuela desde sus inicios: la transmisión de la cultura específica de la sociedad en la que está inmersa, la ayuda a la integración y adaptación de cada alumno a esa comunidad, por lo que enseñarán las normas, las pautas de conducta, etc., propias de esa sociedad, el desarrollo de destrezas específicas dirigidas al desarrollo profesional, y la aportación de la posibilidad de convivir con los iguales y con los adultos en espacios comunes reglamentados. Por ello, la escuela no es lugar donde exclusivamente se enseñan valores en materias específicas como ética o religión, sino que también es un lugar propicio para una transmisión continua, permanente, y duradera de los mismos por parte de toda la comunidad educativa.

Uno de los actores más significativos en el proceso de enseñanza-aprendizaje de valores son los docentes que, por medio de estrategias conductuales, actitudinales, pedagógicas, didácticas y metodológicas, van aproximando al niño a una práctica de valores. Rogers (1969) reconoce la importancia de los valores de los maestros, de la comprensión empática, la realidad, la aceptación y la confianza en la creación de un clima positivo en el aula para fomentar el aprendizaje de los estudiantes. Por ello, Klaassen y Maslovaty (2010) en un estudio realizado con docentes, conciben que el valor moral en el docente "requiere que sea capaz y esté dispuesto a defender en público los propios principios. El valor moral implica atreverse a un cambio ya una "lucha" por los propios principios" (p. 13).

\section{Metodología}

El proyecto: La escuela en la formación de valores comenzó con el objetivo específico de fomentar los valores de manera integral, para fortalecer la unión y atención entre estudiantes, padres de familia y docentes y se enmarcó en unos de los objetivos del Plan Nacional del Buen Vivir: mejorar la calidad de vida de la población.

Esta propuesta se caracterizó por ser esencial, multidisciplinaria e integradora, que hizo partícipe a la comunidad del acervo científico, tecnológico y humanístico y se realizó un diagnóstico previo de necesidades de la colectividad. Se utilizó una metodología activa conformada por una serie de talleres de formación de formadores en temas de valores que los estudiantes dictan en diferentes colegios del país durante un semestre. Esta propuesta permitió formar en los estudiantes de las instituciones educativas participantes un espíritu crítico capaz de analizar con objetividad los problemas sociales de tal manera que pongan sus conocimientos al servicio de los demás desde la visión del humanismo de Cristo. Se desarrollaron competencias y habilidades para la vida profesional, permitió al estudiante integrar de forma natural un elevado sentido de la ética y la vivencia concreta de valores en forma de virtudes, que enriquezcan su actuar profesional para que aporte activamente al desarrollo humano y social de su entorno.

El crear un espacio de apoyo y reflexión que responda a las necesidades prioritarias desde una perspectiva integral de "Educación, Familia y Escuela", con el fin de consolidar valores y actitudes positivas en estudiantes, padres de familia y docentes de las instituciones educativas de los sectores: Santa Rosa, Guayaquil, Quito Bahía de Caraques, Cuenca, Ibarra y Ambato, fue parte de la motivación que dieron inicio a comenzar a identificar las necesidades y requerimientos de los centros educativos, buscando integrar 
conocimientos y conceptualizar principios fundamentales de educación familiar como también una adecuada formación y capacitación para garantizar de esta manera la formación integral de los estudiantes, padres de familia y docentes.

Un primer proceso que se realizó, fue la identificación de necesidades en los centros educativos en relación a la vivencia de valores, utilizando fichas de observación y entrevista a los estudiantes, docentes y padres de familia en diferentes actividades académicas. Con la observación de la cotidianidad de la vida estudiantil en los ocho establecimientos educativos: Unidad educativa PCEI "Juan León Mera", Unidad Educativa "Baltasara Calderón de Rocafuerte", Unidad Educativa "Santa Teresita", Escuela de educación General Básica "Martha Bucaram de Roldós", Unidad Educativa "José F. Hidalgo", Unidad Educativa de la Misión Idente "Pérez Pallares" y Unidad Educativa Particular "Oviedo" los estudiantes de la UTPL del componente Pasantías Pre -Profesionales y de Vinculación con la Colectividad (Practicum 3.2) a través del proyecto de vinculación con la colectividad "La escuela en la formación de Valores", pudieron encontrar que existen una falta de responsabilidad de la familia y control en el uso exagerado de medios electrónicos, se evidencia impuntualidad de los estudiantes, un comportamiento inadecuado, falta de disciplina, manifiestan actitudes poco asertivas en la comunicación, la convivencia en el aula no es armónica, se evidencia poco interés y aburrimiento, que perjudica el trabajo ordenado y adecuado de los docentes en el salón de clases.

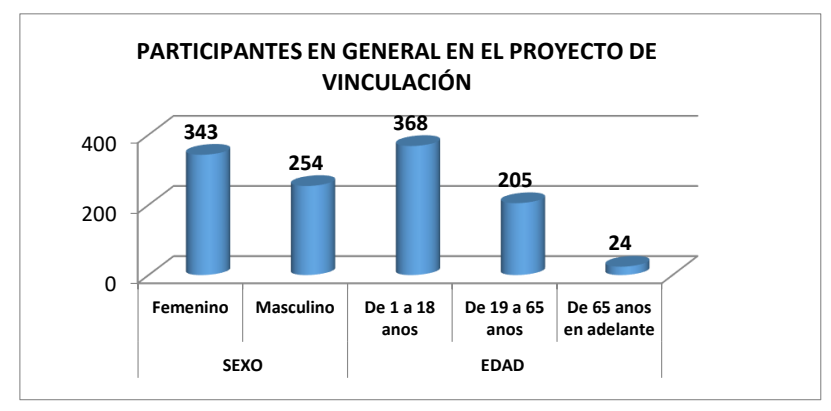

Figura 1. Participantes

Fuente: Autores
Por lo tanto, se consideró pertinente abordar estos temas relacionados a los valores, según manifestaba un pedagogo cristiano: "Es mejor prevenir que reprimir, el error se previene con la instrucción y el vicio inculcando buenos hábitos (San Juan Bosco). Para lograrlo se propone realizar cinco talleres de forma dinámica mediante recursos didácticos, técnicas de motivación, descubrimiento, trabajo grupal, se elaboró una planificación de talleres con el objetivo específico de brindar estrategias pedagógicas como herramientas de orientación a padres de familia y docentes en el rol fundamental de educar a los hijos, a partir del análisis de los distintos aspectos referentes al ambiente familiar, educativo y social, los mismos que tenían una duración de 60 minutos con los estudiantes, 90 minutos con los padres de familia y 60 minutos con los docentes. También se diseñó estrategias de comunicación aplicables entre padres, docentes y estudiantes como medio de prevención en el campo familiar y educativo.

Luego se socializó el proyecto con los rectores de los establecimientos educativos involucrados para su respectiva revisión y aprobación.

Algunos de los temas tratados fueron: puntualidad, orden, convivencia familiar, salud, tecnológico, amor, comunicación asertiva, respecto y puntualidad; los cuales tienen un objetivo y que en conjunto intentan motivar a la vivencia de los valores. Cada una de las actividades propuesta en los mismo se ejecutó con facilidad y creatividad, los estudiantes concretaron lo aprendido a través de carteles, dramatizaciones trípticos, sopa de letras y diálogos familiares, se utilizaron recursos humanos, tecnológicos y materiales.

\section{Resultados}

Es importante tomar en cuenta los aspectos positivos Dauber y Epstein (1993), que resaltan en la conducta de los estudiantes y su relación interpersonal con padres de familia y docentes con la finalidad de obtener como impacto los siguientes indicadores obtenidos de la aplicación de un instrumento con la finalidad de conocer algunos aspectos:

Tabla 1 Actitud del estudiante en la institución educativa

\begin{tabular}{l|ccc}
\hline \multicolumn{1}{c}{ ITEM } & IE1 & IE2 & IE3 \\
\hline Muy Feliz & 58 & 53 & 45 \\
Más o menos & 40 & 39 & 30 \\
Triste & 16 & 3 & 6 \\
TOTAL & $\mathbf{1 1 4}$ & $\mathbf{9 5}$ & $\mathbf{8 1}$
\end{tabular}

Fuente: Autores 
En esta tabla 1 se puede evidenciar que el $53.8 \%$ de los estudiantes encuestados se encuentran con actitud positiva y adecuada en los salones de clase, tomando en cuenta que los estudiantes cuentan con rendimiento escolar medio.
Es por ello importante señalizar que educar en valores es un proceso constante por lo que implica implementar estrategias que lleven a un trabajo continúo en la trilogía familia, escuela y sociedad.

Tabla 2 Convivencia escolar

\begin{tabular}{|c|c|c|c|c|c|c|c|c|c|}
\hline & \multicolumn{3}{|c|}{ IE1 } & \multicolumn{3}{|c|}{ IE2 } & \multicolumn{3}{|c|}{ IE3 } \\
\hline Item & $\mathbf{S i}$ & No & $\begin{array}{l}\text { Más o } \\
\text { Menos }\end{array}$ & $\mathbf{S i}$ & No & $\begin{array}{l}\text { Más o } \\
\text { Menos }\end{array}$ & $\mathbf{S i}$ & No & $\begin{array}{l}\text { Más o } \\
\text { Menos }\end{array}$ \\
\hline Conoce el reglamento escolar & 60 & 12 & 42 & 50 & 17 & 28 & 29 & 10 & 42 \\
\hline Aplica las normas del reglamento escolar & 71 & 3 & 40 & 68 & 9 & 18 & 64 & 5 & 12 \\
\hline $\begin{array}{l}\text { Profesores conversan y ayudan a los alumnos des- } \\
\text { ordenados }\end{array}$ & 30 & 0 & 84 & 77 & 15 & 3 & 47 & 6 & 28 \\
\hline $\begin{array}{l}\text { Existen espacios para reflexionar en situaciones de } \\
\text { conflicto }\end{array}$ & 41 & 6 & 67 & 57 & 9 & 29 & 15 & 20 & 46 \\
\hline Actividades recreativas fuera del salón de clases & 48 & 8 & 58 & 65 & 13 & 17 & 20 & 11 & 50 \\
\hline Alguna vez te ha golpeado algún compañero & 38 & 10 & 66 & 12 & 76 & 7 & 30 & 40 & 11 \\
\hline Te has sentido agredido por algún docente & 43 & 5 & 66 & 4 & 85 & 6 & 25 & 40 & 16 \\
\hline Alguna vez han insultado a alguna persona & 71 & 3 & 40 & 11 & 70 & 14 & 15 & 20 & 46 \\
\hline
\end{tabular}

Fuente: Autores

En las instituciones educativas con la convivencia escolar tratan de construir adecuadas relaciones entre las personas de una comunidad, sustentada en el respeto mutuo y en la solidaridad recíproca, expresada en la interrelación armoniosa y sin violencia entre los docentes, estudiantes y padres de familia. Más del $50 \%$ de los estudiantes participantes en los talleres manifiestan conocer el reglamento escolar y aplicar las normas del mismo. El $40 \%$ no tiene muy claro los espacios creados para el diálogo con los docentes cuando existe desorden o situaciones de conflicto, es así que se comprueba la enorme preocupación de los directivos y maestros para mejorar la disciplina y el involucramiento de los padres de familia en el rol de la formación de los estudiantes.

Es por ello que la familia en la educación, parte de la cual viene dada por la autoridad paterna, que tiene el propósito de transmitir valores de responsabilidad y afectividad, pero con el señalamiento al mismo tiempo de una serie de reglas y límites que proporcionen un equilibrio en la toma de decisiones, conjugando así una interacción recíproca entre padres e hijos así lo confirman varios estudios (Baumrind, 1989; Musitu y Gracia, 2004; Valdivia , 2008; Martín- Quintana, Byme, Ruiz, y Suárez, 2009; Fioravanti , 2010; Rodrigo y Palacios, 2010; Doménech y Cabero, 2011; Sallés y Ger; 2011).

Se puede también observar en la tabla 2, que el $25 \%$ de los estudiantes han sentido algún momento agredido por sus profesores dentro o fuera las aulas: verbal, emocional y físico. Por lo cual se justifica una vez más la intervención de los talleres relacionados a la vivencia de valores.

Tabla 3 Relacionadas en el hogar

\begin{tabular}{|c|c|c|c|c|c|c|c|c|c|c|c|c|}
\hline \multirow[b]{2}{*}{ Item } & \multicolumn{4}{|c|}{ IE1 } & \multicolumn{4}{|c|}{ IE2 } & \multicolumn{4}{|c|}{ IE3 } \\
\hline & Mamá & $\begin{array}{l}\text { Padres } \\
\text { y her- } \\
\text { manos }\end{array}$ & $\begin{array}{l}\text { Otros } \\
\text { fami- } \\
\text { liares }\end{array}$ & Nadie & Mamá & $\begin{array}{l}\text { Padres } \\
\text { y her- } \\
\text { manos }\end{array}$ & $\begin{array}{l}\text { Otros } \\
\text { fami- } \\
\text { liares }\end{array}$ & Nadie & Mamá & $\begin{array}{l}\text { Padres } \\
\text { y her- } \\
\text { manos }\end{array}$ & $\begin{array}{l}\text { Otros } \\
\text { fami- } \\
\text { liares }\end{array}$ & Nadie \\
\hline Vive contigo & 21 & 82 & 11 & 0 & 18 & 68 & 9 & 0 & 30 & 26 & 25 & 0 \\
\hline $\begin{array}{l}\text { Regresa a tu casa, con quien te } \\
\text { encuentras }\end{array}$ & 47 & 30 & 31 & 6 & 39 & 25 & 26 & 5 & 26 & 18 & 32 & 5 \\
\hline $\begin{array}{l}\text { Ayuda a realizar tus tareas es- } \\
\text { colares }\end{array}$ & 52 & 20 & 32 & 10 & 43 & 33 & 14 & 5 & 45 & 10 & 21 & 5 \\
\hline $\begin{array}{l}\text { Tus padres revisan tus cuader- } \\
\text { nos y agenda escolar }\end{array}$ & 42 & 18 & 42 & 10 & 34 & 34 & 22 & 5 & 43 & 27 & 10 & 1 \\
\hline
\end{tabular}

Fuente: Autores

Se comprueba que la enseñanza en niños y adolescentes de los valores en un alto nivel de incidencia se desarrolla en la familia promovida por el ser - hacer de sus progenitores, sin dejar a un lado los factores externos de medios de comunicación y sociedad en general. Más tarde en la escuela estos valores deberán ser fortalecidos por los maestros.
En la encuesta aplicada a los estudiantes se puede obsrvar que el $32.8 \%$ viven con otros familiares: abuelos, tíos o primos; y el $5.5 \%$ se encuentran solos cuando regresan a su hogar, ya sea porque sus padres se encuentran trabajando o tienen problemas de vicios; provocando que los mismos no tengan con quién dialogar sus problemas, inquietudes y reciban orientación sobre sus tareas escolares. 
Sus docentes son los primeros que motivan a los representantes de los estudiantes a cumplir adecuadamente el rol de tutores con los estudiantes y les explica sobre la importancia que tiene la influencia de la familia en el proceso de desarrollo de los jóvenes, la misma que se evidencia en las etapas evolutivas como: agresividad vs no agresividad, logros escolares vs fracaso, motivación $v s$ desmotivación entre otros, y a la vez, informar que estas características propias adquiridas en la familia se relacionan con el mundo externo como son la escuela y la sociedad.

\section{Conclusiones}

Se cumplió el objetivo fundamental que perseguía este proyecto, el orientar el desarrollo científico, tecnológico y humanístico, en base al conocimiento socio-político y económico para solucionar problemas en los establecimientos educativos: básicos, estructurales, de género en relación a la vivencia de valores, partiendo del reto que se presenta a la humanidad como es el no solo trabajar por la prevención de conflictos armados, sino encontrar los medios que posibiliten el cambio definitivo de valores, actitudes, y comportamientos que se traduzcan en paz, equidad, igualdad, libertad, diálogo intercultural, respeto y comprensión mutua, valoración de la diversidad y de las diferencias, tolerancia, y la solución no violenta de los conflictos desde un enfoque de derechos humanos y de ciudadanía, principios democráticos sobre los cuales se sustenta el desarrollo sostenible.

Se logró promover la transferencia e intercambio de aprendizaje entre los miembros de la universidad y la comunidad, acercando a los estudiantes y docentes a un contexto real a través de talleres que generen impacto positivo, en especial en grupos vulnerables de la sociedad.

Como otro resultado se evidencia que la vivencia de valores está en estrecha relación con tres aspectos: convivencia escolar, las relaciones con el docente y en el hogar. El educar en valores es un proceso constante por lo que implica implementar estrategias que lleven a un trabajo continuo en la trilogía familia, escuela y sociedad.

El proyecto de vinculación desarrolló una sinergia entre docentes universitarios y estudiantes de la Modalidad Abierta, además se logró incrementar un aprendizaje colaborativo y el refuerzo de algunas competencias como docentes. Es fundamental reafirmar que un aprendizaje significativo se logra implicando a los alumnos en la construcción del mismo. Se ha realizado la campaña de concienciación en los valores dirigida a todos los estudiantes del establecimiento en donde ellos realizan su práctica docente durante el periodo marzo 2017 a marzo del 2018.

Un aprendizaje transversal es la inclusión de valores en las aulas. La disciplina es muy importante para fomentar un clima de aula positivo y que por ende se logren unas buenas relaciones dentro del aula y que la convivencia llegue a ser armónica, para el logro de este objetivo se ha fomentado en los estudiantes de las instituciones educativas los siguientes valores: amor, puntualidad, comunicación y respeto.

Por último, se consiguió concientizar a los estudiantes, docentes y padres de familia sobre la importancia de poner en práctica los valores que lleven a crear un ambiente adecuado donde se facilite el trabajo en equipo, se reduzcan los conflictos y se potencien la paz mundial y el bienestar general de la humanidad.

\section{Referencias Bibliográficas}

Gordillo, M. (2015). Educar es mucho más que ense nar: es humanizar. Descargado de http://www.oei.es/historico/ divulgacioncientifica/?Mariano -Martin-Gordillo-

Jackson, P. W. (1992). Conceptions of curriculum and curriculum specialists. Handbook of research on curriculum, 3, 40.

Klaassen, C. A., y Maslovaty, N. (2010). Moral courage and the normative professionalism of teachers. Rotterdam: Sense.

Ortiz, J. (2003). La educación en valores y su práctica en el aula. Tendencias pedagógicas, 8, 69.

Populorum Progressio. (1965). Discurso en las naciones unidas. Descargado de http://www.vatican .va/roman_curia/congregations/ ccatheduc/documents/rc_con_cca (4 de octubre de 1965)

Rogers, C. R. (1969). Freedom to learn: A view of what education might become. Columbus, OH: C.E.: Merrill.

Rokeach, M. (1973). The nature of human values. En N. N. Y. F. Press (Ed.), Free press.

Ruiz Corbella, M. (2003). Educación moral: aprender a ser, aprender a convivir. Barcelona: Ariel.

Suárez, R. (2008). Artículo educación condición de humanización. Centro Editorial Universidad de Caldas Manizales Colombia S. A.

Recibido: 24 de mayo de 2019

Aceptado: 15 de julio de 2019 